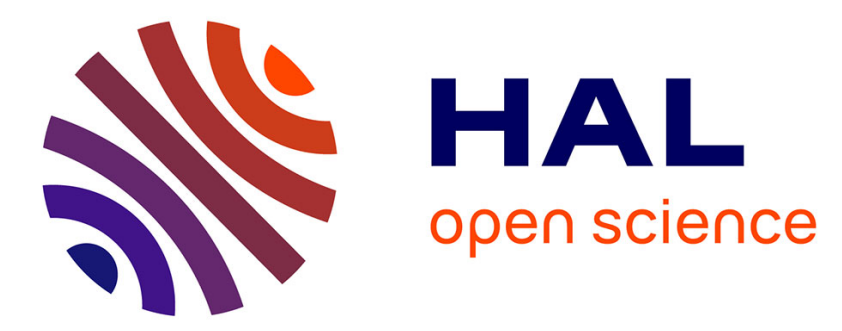

\title{
Formaldehyde passive sampler using an optical chemical sensor: how to limit the humidity interference
}

\author{
Jane Vignau-Laulhere, Pierre Mocho, Hervé Plaisance, K. Raulin-Woznica,
} Thu-Hoa Tran-Thi, V. Desauziers

\section{- To cite this version: \\ Jane Vignau-Laulhere, Pierre Mocho, Hervé Plaisance, K. Raulin-Woznica, Thu-Hoa Tran-Thi, et al.. Formaldehyde passive sampler using an optical chemical sensor: how to limit the humidity interference. Analytical and Bioanalytical Chemistry, 2017, 409, pp.6245-6252. 10.1007/s00216-017-0568-3 . cea- 01575440}

HAL Id: cea-01575440

https://hal-cea.archives-ouvertes.fr/cea-01575440

Submitted on 20 Feb 2020

HAL is a multi-disciplinary open access archive for the deposit and dissemination of scientific research documents, whether they are published or not. The documents may come from teaching and research institutions in France or abroad, or from public or private research centers.
L'archive ouverte pluridisciplinaire HAL, est destinée au dépôt et à la diffusion de documents scientifiques de niveau recherche, publiés ou non, émanant des établissements d'enseignement et de recherche français ou étrangers, des laboratoires publics ou privés. 


\title{
Formaldehyde passive sampler using an optical chemical sensor: how to limit the humidity interference
}

\author{
Jane Vignau-Laulhere ${ }^{1,2}$ - Pierre Mocho $^{3}$ - Hervé Plaisance ${ }^{2}$. \\ Katarzyna Raulin-Woznica ${ }^{1}$. Thu-Hoa Tran-Thi ${ }^{4} \cdot$ Valérie Desauzier
}

\begin{abstract}
The behaviour of a new formaldehyde diffusive sampler using an optical chemical sensor with respect to high humidity conditions is examined in controlled atmospheres. Five prototypes of the radial diffusion sampler having the same chemical sensor and different designs were tested. In addition, a set of experiments were performed on the chemical sensor to characterise its efficiency of trapping water vapour in the absence and in the presence of the reactants, Fluoral-P and formaldehyde. Differences in humidity interference between the five diffusive sampler prototypes were studied and discussed. From all the results obtained, it was shown that the prototype LDE1.4 combining a small diffusion slot, a reduced internal volume and a sensor shifted upwards from the diffusion slot provided formaldehyde measurements least affected by humidity up to $80 \% \mathrm{RH}$ at $20^{\circ} \mathrm{C}$. This new type of diffusive sampler with on-site direct reading is intended to ultimately replace conventional passive samplers with DNPH requiring offset laboratory analyses.
\end{abstract}

Keywords Radial diffusive sampler · Gas sensor · Fluoral-P . Exposure chamber $\cdot$ Formaldehyde

Valérie Desauziers

valerie.desauziers@mines-ales.fr

Ethera, 629 rue Charles de Gaulle, 38920 Crolles, France

2 Pôle RIME-C2MA, Ecole des Mines d'Alès, Hélioparc, 2 Avenue Pierre Angot, 64053 Pau Cedex 9, France

3 Laboratoire Thermique Energétique et Procédés, Université de Pau et des Pays de l'Adour, BP 7511, 64053 Pau, France

4 NIMBE, CEA, CNRS, Université Paris-Saclay, CEA-Saclay, 91191 Gif sur Yvette Cedex, France

\section{Introduction}

Among indoor air pollutants, formaldehyde is one of the most abundant volatile organic compounds (VOC) in indoor air [1] and may cause acute health effects. At low concentrations in the parts per billion range, it can induce eye and respiratory irritations [2,3] and asthmatic symptoms under chronical exposure [4]. Long-term exposure to high concentration of formaldehyde (ppm range) in working places was strongly suspected to be the cause of nasopharyngeal cancers, which led the International Agency for Research on Cancer to upgrade its classification from probably carcinogenic to carcinogenic to humans $[3,5]$. The indoor levels of formaldehyde in homes, schools and work offices are typically of the order 1$100 \mathrm{ppb}$ but can sometimes reach higher values when the emissions of indoor sources are high and the ventilation is deficient [6].

Up to now, the standard methods of measuring formaldehyde indoors are the active (NF ISO 16000-3, 2002) and passive (NF ISO 16000-4, 2006) samplings using 2,4dinitrophenylhydrazine as trapping reagent. The minimum sampling time is $30 \mathrm{~min}$, and these methods require a second step of analysis in laboratory of the hydrazones formed by reaction of 2,4-dinitrophenylhydrazine with the aldehydes present in the probed atmosphere.

Few methods are able to supply on-site formaldehyde measurements with the requested selectivity and sensitivity for indoor air. Three commercialised instruments with active sampling meet the following requirements: AL4021 from AEROLASER $® ~[7,8]$, FM-801 from GrayWolf based on a colorimetric reaction and 4160-1000b from INTERSCAN® based on an electrochemical reaction [9]. AEROLASER and GrayWolf use the same well-established Hantzch reaction starting with acetylacetone and ammonia and detect the final product 3,5-diacetyl-1,4-dihydrolutidine (DDL), issued from 
the reaction with formaldehyde. AEROLASER uses liquid phase reactions at low temperature and detects DDL via fluorescence with a detection limit of $0.1 \mathrm{\mu g} \mathrm{m}^{-3}$ but is not suitable for a multi-site and large campaign of measurements because of its cost [9]. GrayWolf device using Vycor glass sheets impregnated with the reactants is portable and easy to use, but its detection limit is $25 \mu \mathrm{g} \mathrm{m}^{-3}$. For multi-site campaign of measurements, a passive sampler with a direct on-site reading would be an interesting technical advance. More recently, ETHERA had proposed a solid-state colorimetric sensor as trapping media inserted in a diffusive sampler. The sensing reagent, proposed by ETHERA is Fluoral-P or 4-amino-3penten-2-one, an intermediate species involved in the wellknown Hantzch reaction [10] and discovered by Compton and Purdy [11]. The reaction of Fluoral-P with formaldehyde also leads to DDL whose concentration can be measured by fluorescence or absorption spectrometry [12]. Although the fluorescence technique offers a higher sensitivity, absorption is preferred for field instruments because it is less expensive than fluorescence. The nanoporous monoliths doped with Fluoral-P were produced via a sol-gel method [13]. This synthesis method allows the tailoring of the pore size to trap the probe molecules, which are homogeneously distributed. The advantages over impregnated porous solids are twofold: there is no leaching of the reactant and high concentration of the probe molecules can be obtained due to the shrinkage process when going from the sol to the dried monolith. However, many authors reported that chemical sensors based on Fluoral-P and formaldehyde reaction can suffer from humidity interferences [14, 15]. Dagnelie et al. [14] report that Fluoral-P in various nanoporous thin films can be hydrolysed by gaseous water accumulated in the pores at high concentration and show that the amount of trapped water varies accordingly to the hydrophobicity of the support matrix. However, it should be noted that Dagnelie's matrices display an acidic intrapore $\mathrm{pH}$ due to the presence of residual $\mathrm{Si}-\mathrm{OH}$ groups, while hybrid matrices functionalised with aminopropyl groups are less acidic. Since water vapour appears as the main interfering gas for the Fluoral-P/formaldehyde reaction, it is important to probe its role with the aim of limiting or even suppressing its interference.

In this paper, the objective is to determine among five prototypes of ETHERA the best suited for the measurement of formaldehyde concentration in high humidity conditions. To this end, various steps were needed. The first one is to characterise the response of sensor to formaldehyde for the five prototypes of radial diffusive sampler displaying different geometrical characteristics (internal volume, membrane slot, path from the membrane to the nanoporous sensor) and its ability to trap water. Then, the influence of humidity on the sensor response for the five diffusive samplers exposed to formaldehyde was studied with the measurement of the water uptake by the sensor. These experiments aim at understanding the role of water molecules in the Fluoral-P/formaldehyde reaction and to discriminate the geometrical features of the diffusive sampler which can efficiently limit the humidity interference.

\section{Materials and methods}

\section{Nanoporous sensor doped with Fluoral-P}

The sensor is a parallelepipedal, transparent and nanoporous monolith with a sampling surface of $125.4 \mathrm{~mm}^{2}$. The nanoporous monolith was produced via the Sol-Gel process [16], using as precursors tetramethyl orthosilicate (TMOS) and (3-aminopropyl) triethoxysilane (APTES) and Fluoral-P (98\% pure) as reagent [13]. Briefly, TMOS, APTES, a methanolic solution of Fluoral-P $\left(1.06 \mathrm{~mol} \mathrm{~L}^{-1}\right)$ and water, in respective $0.97 / 0.03 / 5 / 4 \mathrm{~mol}$ proportion are mixed together stepwise and the mixture is continuously stirred. The concentration of Fluoral-P in the final mixture is $0.5 \mathrm{~mol} \mathrm{~L}^{-1}$. Before addition of water, the mixture is cooled down to $-20^{\circ} \mathrm{C}$. The final mixture is then poured into a mould where gelation occurs, and the material is dried under inert gas during 1 week. The final dimensions of the monolith after shrinkage is $4.8 \times 1.9 \times 8 \mathrm{~mm}$, and the Fluoral-P concentration is $3.2 \mathrm{~mol} \mathrm{~cm}^{-3}$. The porosity parameters, specific adsorption surface area and pore size distribution were determined via collecting adsorption isotherms of $\mathrm{N}_{2}$ at liquid nitrogen temperature with the Quantachrome Autosorb 1.The sensor displays a large specific surface area of adsorption, $870 \pm 60 \mathrm{~m}^{2} \mathrm{~g}^{-1}$, a total pore volume of $0.78 \pm 0.03 \mathrm{~cm}^{3} \mathrm{~g}^{-1}$ and a pore size distribution centred around $32 \pm 13 \AA$. Undoped matrices were also produced to compare the water trapping efficiency of both undoped and doped matrices.

The selective detection of formaldehyde is based on the absorbance measurement of a coloured compound, 3,5diacetyl-1,4-dihydrolutidine (DDL) formed by reaction between formaldehyde and Fluoral-P [17]. While Fluoral-P absorbs uniquely the UV light, DDL display a wide absorption band in the UV-visible region peaking at $410 \mathrm{~nm}$ (Fig. 1).

The produced DDL is quantified by a direct absorbance measurement through the monolith at $420 \mathrm{~nm}$ by means of an optical reading module comprising a LED source centred at $420 \mathrm{~nm}$. The absorbance change is proportional to the DDL amount, which is directly linked to the formaldehyde concentration [18].

\section{ETHERA ${ }^{\circledR}$ passive samplers}

The geometry of the five prototypes of diffusive samplers having different geometric characteristics (internal volume, diffusion slot and position of trapping media in the passive sampler) was described in a previous paper [19]. Briefly, the 


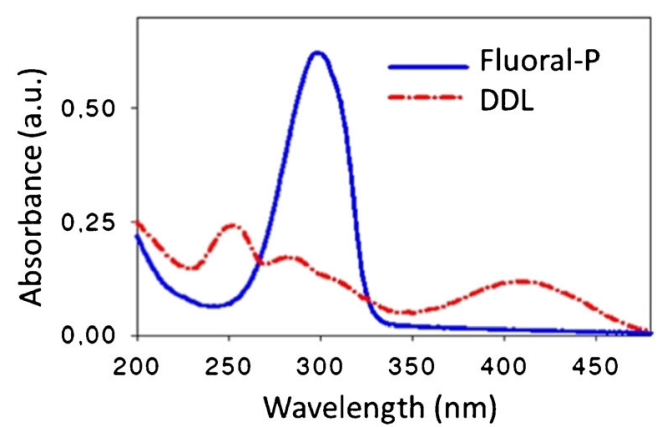

Fig. 1 Absorption spectra of Fluoral-P and DDL in a thin film of nanoporous material

passive samplers (Fig. 2) have a cylindrical shape and are all supplied with the same diffusion membrane. The latter is a micro-porous polyethylene cylinder with a $27.6 \mathrm{~mm}$ external diameter, $10 \mathrm{~mm}$ long, $3.2 \mathrm{~mm}$ wall thickness and a mean radius of $5 \mu \mathrm{m}$ pore size. Previous experiments coupled with numerical simulations based on Fick's laws allowed to estimate the effective diffusion coefficient of formaldehyde through this membrane at $3.50 \times 10^{-6} \mathrm{~m}^{2} \mathrm{~s}^{-1}$ [19]. Two diffusion slots are symmetrically arranged on the outer surface of the membrane. Two widths of diffusion slot were tested, 1.4 and $5.9 \mathrm{~mm}$ corresponding to a membrane exposed external surface of 96 and $404 \mathrm{~mm}^{2}$. Two plastic components hold the membrane and ensure the air tightness. Two different internal volumes of the prototypes are available: the QAI (QAI 1.4, QAI 5.9 and QAI 1.4dep) and LDE (LDE 1.4 and LDE 5.9) samplers display an internal volume of 18.97 and $6.14 \mathrm{~cm}^{3}$, respectively. For the two samplers QAI 1.4 and QAI 5.9, the sensor is positioned at the same height as the diffusion slot whereas for the three samplers LDE 1.4, LDE 5.9 and QAI $1.4 \mathrm{dep}$, the sensor is shifted upwards by $1.2 \mathrm{~cm}$ relatively to the slot position.

In the case of passive sampling using a sensor, the sampled formaldehyde mass cannot be directly assessed. For this purpose, the sensor response noted $K\left(\mathrm{ppb}^{-1} \mathrm{~min}^{-1}\right)$ is defined as follows:

$K=\frac{\Delta \mathrm{Abs}}{C \times t}$

where $\Delta \mathrm{Abs}$ is the absorbance change of the sensor after exposition, $C$ formaldehyde concentration (ppb) and $t$ the exposure time ( $\mathrm{min})$.

In a previous study [19], sampling rates of these five diffusive samplers were experimentally assessed and confirmed by numerical modelling based on Fick's laws. It was shown that the sampling rate increases with the size of the diffusion slot; this effect being more pronounced when the sensor is positioned at the same height than the slot. Sampling rates were assessed to $8.84 \mathrm{~mL} \mathrm{~min}^{-1}$ for LDE $1.4,9.14 \mathrm{~mL} \mathrm{~min}^{-1}$ for QAI 1.4dep, $10.03 \mathrm{~mL} \mathrm{~min}^{-1}$ for LDE 5.9, $11.76 \mathrm{~mL} \mathrm{~min}^{-1}$ for QAI 1.4 and $17.44 \mathrm{~mL} \mathrm{~min}^{-1}$ for QAI 5.9.

\section{Exposure experiments}

To evaluate the influence of humidity on the sensor response to formaldehyde, the five prototypes of diffusive samplers were tested in an exposure chamber controlled in formaldehyde concentration $([\mathrm{HCHO}])$, temperature $\left(T^{\circ}\right)$ and relative humidity (RH).

The experimental device consists of a module for generation of standard atmospheres, a glass exposure chamber of $50.9 \mathrm{~L}$ in which the diffusive samplers are placed and probes and an air sampling device to monitor and control the temperature, relative humidity and formaldehyde concentration. It was already described in detail in a previous article [20]. Diffusive samplers were tested under various atmospheres controlled in formaldehyde concentration of about $30 \mathrm{ppb}$ at $20{ }^{\circ} \mathrm{C}$ and relative humidity (from 20 to $80 \%$ ). The sampling duration chosen is 4.5 days to meet the requirements for formaldehyde monitoring protocol in France in public buildings receiving vulnerable people [21]. The sensor absorbance was measured before and after each sampling period by means of optical reading module.

The formaldehyde concentration in the exposure chamber is monitored by successive active samplings during 24 and $48 \mathrm{~h}$ with dual-bed 1,2-bis(2-pyridyl)ethylene/DNPH (BPE/
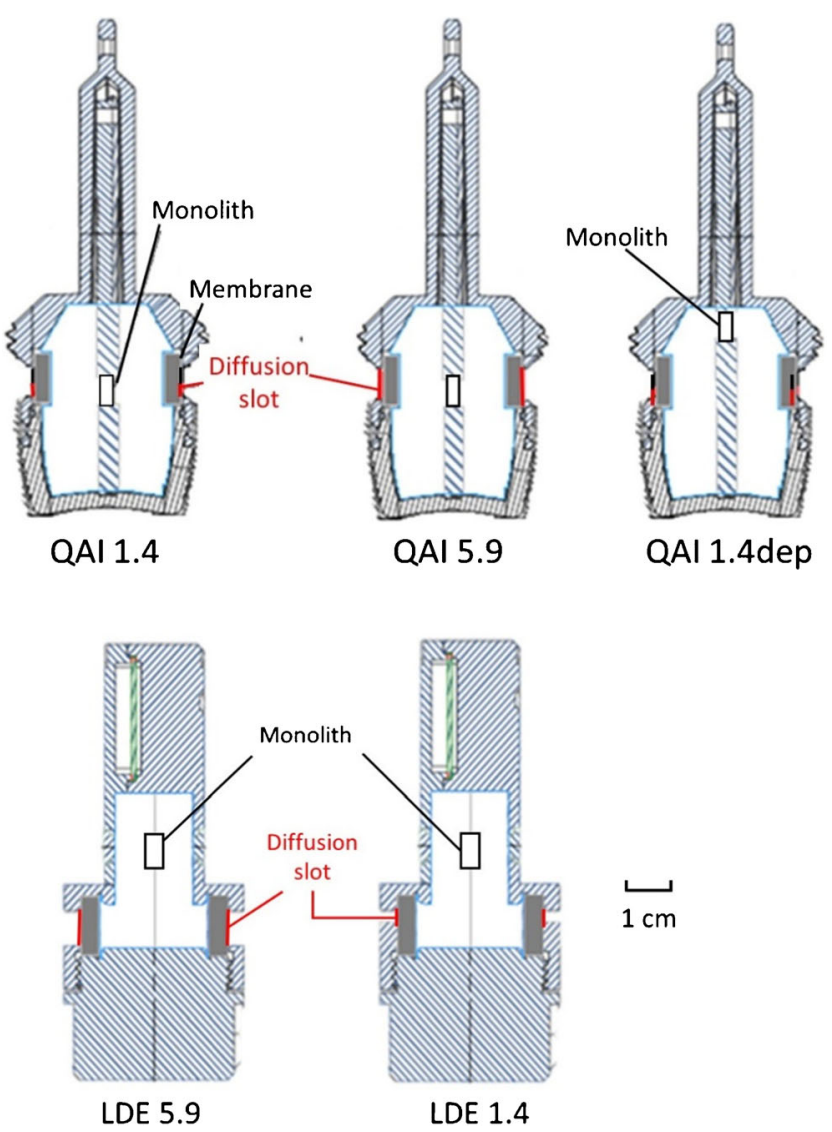

Fig. 2 Cross-sectional plans of the passive samplers 
DNPH) cartridges (Sigma-Aldrich, Saint Louis, MO, USA) developed by Uchiyama [22]. The sampling flow rate was regulated at $50 \mathrm{~mL} \mathrm{~min}{ }^{-1}$ with a mass-flow meter. The protocol followed for the extraction and HPLC analysis of this cartridge was given in Vignau et al. [20].

\section{Results and discussion}

\section{Influence of relative humidity on the sensor response for the five diffusive samplers}

Sixteen experiments were carried out in the exposure chamber with the following experimental conditions: fixed formaldehyde concentration of $30 \mathrm{ppb}$, fixed temperature of $20^{\circ} \mathrm{C}$ and humidity being varied from 20 to $80 \%$. The sensor response $(K)$ is measured for the five diffusive samplers. All the results are shown in Fig. 3.

Between 20 and $45 \%$ RH, all the passive samplers display similar sensor responses $\left(1 \mathrm{ppb}^{-1} \mathrm{~min}^{-1}<\right.$ $K \times 10^{6}<2 \mathrm{ppb}^{-1} \mathrm{~min}^{-1}$ ) which are not affected by humidity. Above 45\% RH, the sensor response increases and this increase is correlated with the formaldehyde sampling rates in the order $\mathrm{SR}_{\mathrm{LDE}} 1.4<\mathrm{SR}_{\mathrm{QAI}} 1.4 \mathrm{dep}<\mathrm{SR}_{\mathrm{LDE}} 5.9<\mathrm{SR}_{\mathrm{QAI}}$ $1.4<\mathrm{SR}_{\mathrm{QAI}}$ 5.9. These observations suggest that the reaction between Fluoral-P and formaldehyde improves in the presence of water molecules.

Since the nanoporous sensor could act as a sponge and accumulate water molecules when exposed to humid conditions, it is important to specifically evaluate the efficiency of trapping of water vapour by the nanoporous sensor.

\section{Efficiency of trapping of water vapour by the nanoporous sensor according to the diffusive samplers under high humidity conditions}

In a previous study [19], the mass transfer of water and the maximal water uptake of the sensor for each diffusive sampler were evaluated. It was shown that the rate of water trapping by the sensor as a function of time follows an inverse exponential function. The final mass of trapped water at equilibrium state is quite similar regardless of the passive sampler. For all samplers, the sensor traps about $27 \mathrm{mg}$ of water under high humidity conditions $\left(80 \% \mathrm{RH}\right.$ and $\left.20^{\circ} \mathrm{C}\right)$. Values of the initial water uptake rate $\left(F_{\mathrm{H}_{2} \mathrm{O}}\right)(t<500 \mathrm{~min})$ are $33.7 \mu \mathrm{g} \mathrm{min}{ }^{-1}$ for LDE 1.4, $36.4 \mu \mathrm{g} \mathrm{min}^{-1}$ for QAI 1.4dep, $36.5 \mu \mathrm{g} \mathrm{min}^{-1}$ for LDE 5.9, $43.4 \mu \mathrm{g} \mathrm{min}^{-1}$ for QAI 1.4 and $48.0 \mu \mathrm{g} \mathrm{min}^{-1}$ for QAI 5.9. For the same height of slot, $F_{\mathrm{H}_{2} \mathrm{O}}$ of QAI is higher than that of LDE, confirming the incidence of membrane/ monolith distance on mass transfer observed for formaldehyde. It can be also noted that the initial water uptake rates for the five diffusive samplers follow the same ranking than

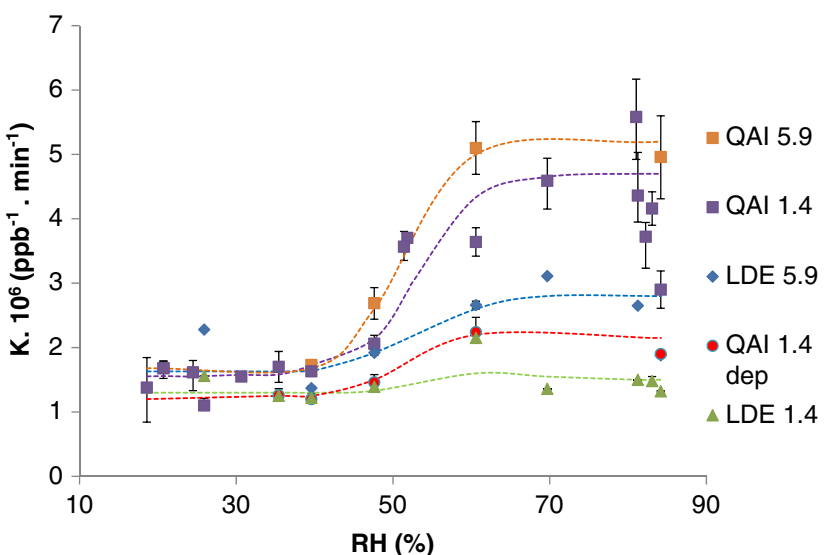

Fig. 3 Sensor responses $\left(\mathrm{K}, \mathrm{ppb}^{-1} \mathrm{~min}^{-1}\right)$ of different passive samplers exposed during $24 \mathrm{~h}$ to $30 \mathrm{ppb}$ of formaldehyde at $20{ }^{\circ} \mathrm{C}$ and relative humidity comprised between 20 and $80 \%$. Bars represent the minimummaximum value range

the formaldehyde sampling rates. This point will be discussed later.

In order to highlight the effect of humidity on the $K$, the relationships between the initial water uptake rate (for $t<500 \mathrm{~min}$ ) by the sensor for each passive sampler and $K$ values found at $80 \% \mathrm{RH}$ was investigated (Fig. 3); since the $K$ is dependent on the sampling rate $\left(\mathrm{SR}_{\mathrm{HCHO}}\right)$, it is necessary to consider a new indicator which is free from the sampling rate contribution. Therefore, the $K / \mathrm{SR}_{\mathrm{HCHO}}$ ratio is chosen as the new indicator.

Figure 4 clearly shows a linear relationship between the sensor response defined as $K / \mathrm{SR}_{\mathrm{HCHO}}$ and the water uptake rate, $F_{\mathrm{H}_{2} \mathrm{O}}$, at least when the relative humidity is $80 \%$. This result shows that the difference in the sensor responses for the five passive samplers at high humidity cannot only be explained by the difference in sampling rates (i.e. formaldehyde flow by diffusion in air). Another effect, which involves the water uptake in the nanoporous sensor must be considered. Since the water uptake rate is dependent on the geometry of the diffusive samplers, our next step is to probe the influence of a wide range of humidity on the sensor response during a long exposure time ( 4.5 days).

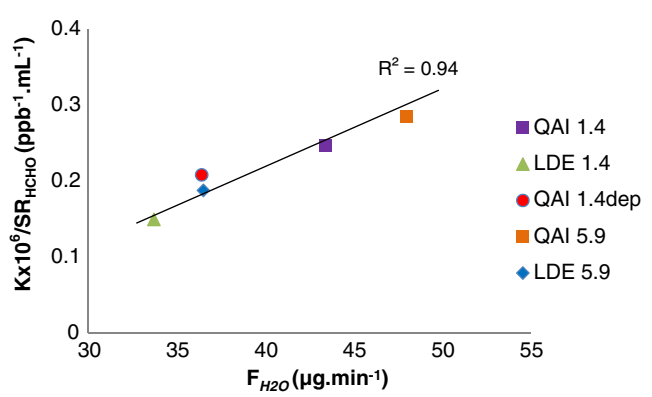

Fig. 4 Ratio of the sensor response $(\mathrm{K})$ on sampling rate $\left(\mathrm{SR}_{\mathrm{HCHO}}\right)$ for the different passive samplers at $30 \mathrm{ppb}$ of formaldehyde, $20^{\circ} \mathrm{C}$ and $80 \%$ $\mathrm{RH}$ versus the initial water uptake rate $(t<500 \mathrm{~min})$ at $80 \% \mathrm{RH}$ 


\section{Effect of humidity on long-term exposure of the passive samplers to formaldehyde}

A series of experiments were carried out to evaluate the humidity effect on the absorbance change during the sampling of formaldehyde during 4.5 days. All the diffusive samplers were exposed to humid mixtures containing $30 \mathrm{ppb}$ of formaldehyde at $20^{\circ} \mathrm{C}$. Four conditions of humidity were chosen 40 , 50,60 and $80 \%$ RH. The absorbance at $420 \mathrm{~nm}$ was measured for each sampler twice a day throughout the test duration. The results are shown in Fig. 5.

The absorbance change at $420 \mathrm{~nm}$ varies linearly with time for all the samplers whatever the relative humidity is. The absorbance change over time increases with increased humidity (see Table 1). This effect is much more pronounced for the samplers which display a high value of the water mass transfer, $F_{\mathrm{H}_{2} \mathrm{O}}$.

In view of the experimental results reported in Figs. 3, 4 and 5, it is noted that small changes of the sampler configuration appear causes major modifications of humidity effect on the sensor response.

Among the passive samplers, LDE 1.4 which displays the smallest volume, the smallest diffusion slot and a sensor shifted relatively to the slot position, appears to be the least affected by humidity. A way used here to limit the humidity interference was to reduce the initial water uptake rate by shifting the sensor with respect to the slot position. This has proven to be effective and lead to an unconventional configuration for a diffusive sampler. The prototype LDE 1.4 appears close to the optimal version of this sampler.

In order to check the influence of water molecules on the reaction rate of Fluoral-P with formaldehyde, additional series of tests in the exposure chamber were carried out during 4.5 days with a same QAI 1.4 passive sampler but under various conditions. Two QAI 1.4 were loaded with a nanoporous sensor, the first one being exposed to humid atmosphere $(\mathrm{RH}=80 \%)$ and the second to humid atmosphere $(\mathrm{RH}=80 \%)$ containing formaldehyde $(30 \mathrm{ppb})$. Two other QAI 1.4 were loaded with a nanoporous monolith free of reactants and exposed in the conditions just described. The results are shown in Fig. 6.

It can be noted that, under high humidity conditions, the monoliths free of reactants do not produce any increase in absorbance at $420 \mathrm{~nm}$ (Fig. 5). In contrast, the absorbance increases at $420 \mathrm{~nm}$ when both Fluoral-P and formaldehyde are present indicates an increase in DDL formation. Therefore, the reaction of Fluoral-P and formaldehyde appears to be strongly affected by the humidity.
Fig. 5 Absorbance change during the 4.5-day sampling of formaldehyde $(30 \mathrm{ppb})$ with the five diffusive samplers placed in the exposure chamber under various humidity conditions (from 40 to $80 \% \mathrm{RH}$ ) at $20{ }^{\circ} \mathrm{C}$ (diamonds $80 \% \mathrm{RH}$, squares $60 \%$ $\mathrm{RH}$, triangles $50 \% \mathrm{RH}$, error marks 40\% RH)
QAI 5.9

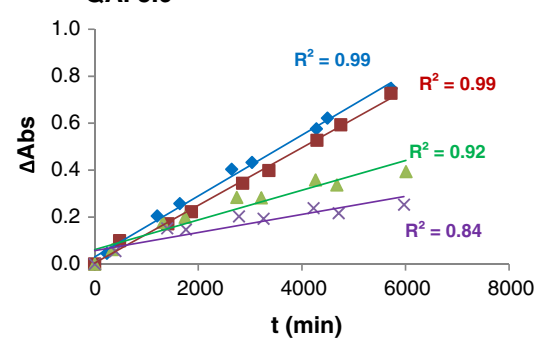

LDE 5.9

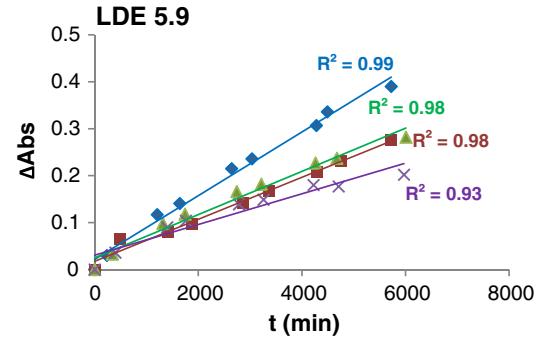

QAI 1.4
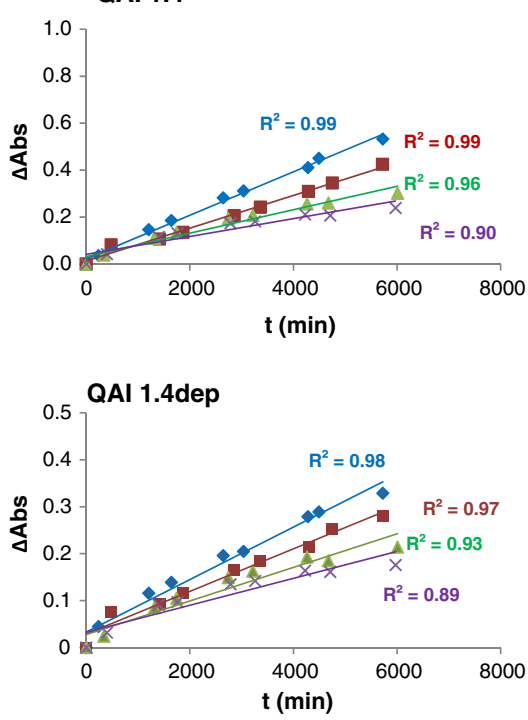

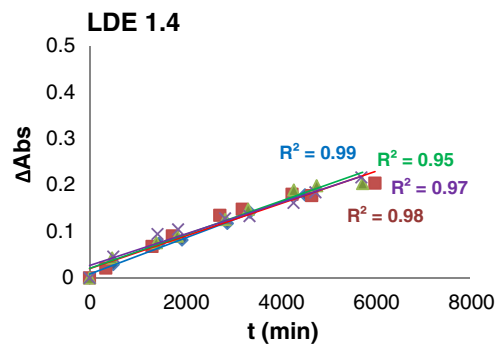


Table 1 Absorbance change over time $\left(\mathrm{min}^{-1}\right)$ and standard deviation of the five passive samplers under various humidity conditions

\begin{tabular}{llllll}
\hline RH (\%) & QAI 5.9 & QAI 1.4 & LDE 5.9 & QAI 1.4dep & LDE 1.4 \\
\hline 80 & $(13.0 \pm 0.4) \times 10^{-5}$ & $(9.3 \pm 0.3) \times 10^{-5}$ & $(6.8 \pm 0.3) \times 10^{-5}$ & $(6.0 \pm 0.3) \times 10^{-5}$ & $(4.2 \pm 0.2) \times 10^{-5}$ \\
60 & $(12.3 \pm 0.3) \times 10^{-5}$ & $(7.0 \pm 0.3) \times 10^{-5}$ & $(4.6 \pm 0.3) \times 10^{-5}$ & $(5.0 \pm 0.3) \times 10^{-5}$ & $(3.5 \pm 0.3) \times 10^{-5}$ \\
50 & $(6.3 \pm 0.7) \times 10^{-5}$ & $(5.0 \pm 0.4) \times 10^{-5}$ & $(4.5 \pm 0.2) \times 10^{-5}$ & $(4.0 \pm 0.4) \times 10^{-5}$ & $(3.5 \pm 0.2) \times 10^{-5}$ \\
40 & $(3.9 \pm 0.6) \times 10^{-5}$ & $(3.8 \pm 0.5) \times 10^{-5}$ & $(3.3 \pm 0.6) \times 10^{-5}$ & $(3.0 \pm 0.4) \times 10^{-5}$ & $(3.1 \pm 0.2) \times 10^{-5}$ \\
\hline
\end{tabular}

The fact that the sensor response follows the same variation trend with humidity than the water uptake was unexpected. When the humidity increases, one would expect a competition between the trapping of water and formaldehyde molecules by the sensor in favour of water. At $20{ }^{\circ} \mathrm{C}$ and $\mathrm{RH}=80 \%$, the water level (13.8 ppm) in the exposure chamber is 460 times higher than the formaldehyde concentration (30 ppb). This competition should have disfavoured the formation of DDL. Moreover, in the presence of water, Fluoral-P can be hydrolysed leading to acetylacetone and ammonia, as shown by Dagnelie et al. [16].

These authors had used Fluoral-P to probe the hydrophobicity of various nanoporous hybrid silicate thin films and matrices displaying in their pores functional groups such as $\mathrm{Si}-\mathrm{OH}, \mathrm{Si}-\mathrm{CH}_{3}$ and $\mathrm{Si}-\mathrm{CH}_{2}-\mathrm{CH}_{2}-\mathrm{CF}_{3}$ in various proportions. These materials doped with Fluoral-P were exposed under static and dynamical modes of exposure to humidified atmospheres $(\mathrm{RH}=100 \%)$. In the presence of humidity, Fluoral-P is hydrolysed leading to acetylacetone and ammonia. By monitoring the absorbance variation of Fluoral-P at $300 \mathrm{~nm}$, they showed that the rate of hydrolysis decreases with increased hydrophobicity of the matrices. More importantly, these authors pointed out that the mass transfer of molecules from the bulk gas phase to the porous surface through a stagnant gas layer is very sensitive to the aerodynamics of the gas flow. A dynamical flow could ease the diffusion of the molecules into the material and this dynamical effect would be independent of the nature of the material, thus explaining the small difference between the less and most hydrophobic materials in stopping the water permeation under a dynamical regime. On the contrary, under static conditions of exposure to humidity ( $\mathrm{RH}=100 \%$ ), the hydrolysis of Fluoral-P is strongly slowed down by a factor 2 to 50 for the less and most hydrophobic matrices, respectively.

Note that the matrices studied by Dagnelie et al. display an acidic intrapore $\mathrm{pH}$ due to the residual acidic $\mathrm{Si}-\mathrm{OH}$ groups, which can favour the Fluoral-P hydrolysis. In contrast, the pores of the present nanoporous sensor are functionalised with $\mathrm{Si}-\mathrm{OH}$ and $\mathrm{Si}-\left(\mathrm{CH}_{2}\right)_{3} \mathrm{NH}_{2}$ groups. Though, its capacity to trap water vapour can still be high, due to the presence of both residual $\mathrm{Si}-\mathrm{OH}$ groups and $\mathrm{Si}-\left(\mathrm{CH}_{2}\right)_{3} \mathrm{NH}_{2}$ groups, the intrapore $\mathrm{pH}$ of the sensor is expected to be less acidic than that of the matrices of Dagnelie.
The intrapore $\mathrm{pH}$ is expected to play an important role both in the hydrolysis of Fluoral-P and in reaction rate of Fluoral-P with formaldehyde. While hydrolysis of Fluoral-P occurs below $\mathrm{pH}=4$, the Fluoral- $\mathrm{P} / \mathrm{CH}_{2} \mathrm{O}$ reaction leading to DDL is catalysed in acidic media and is optimal for $2.5<\mathrm{pH}<4.5$. With $\mathrm{pH}>5$, both reactions slow down. These two reactions are in competition and can only be effective in the presence of water.

The intrapore $\mathrm{pH}$ of matrices of TMOS with $\mathrm{Si}-\mathrm{OH}$ and hybrid TMOS/APTES with various proportions of $\mathrm{Si}-\mathrm{OH}$ and $\mathrm{Si}-\left(\mathrm{CH}_{2}\right)_{3} \mathrm{NH}_{2}$ groups was probed by Bamogo et al. [23]. To probe the intrapore $\mathrm{pH}$ change, these authors used bromothymol blue (BTB), a pH-sensitive dye which displays a $\mathrm{p} K_{\mathrm{a}}$ value of 7.1. In aqueous solutions, BBT displays a yellow-orange colour at acidic $\mathrm{pH}=4.65$ and a dark blue colour at basic $\mathrm{pH}=8$. These two colours correspond to the protonated BBT (orange) and deprotonated BBT $(-\mathrm{H}+)$ (blue). In TMOS matrices, BBT always displays the yellow-orange colour independently of the amount of trapped water, thus indicating that the TMOS matrix display an acidic intrapore $\mathrm{pH}$. In hybrid matrices containing $80 \mathrm{M} \%$ of TMOS and $20 \mathrm{M} \%$ of APTES, BBT always displays a dark blue colour independently of the amount of trapped water. Therefore this matrix displays a basic intrapore $\mathrm{pH}$. In a hybrid matrix TMOS/APTES containing $3 \mathrm{M} \%$ of APTES, BBT displays a green colour corresponding to the combination of the

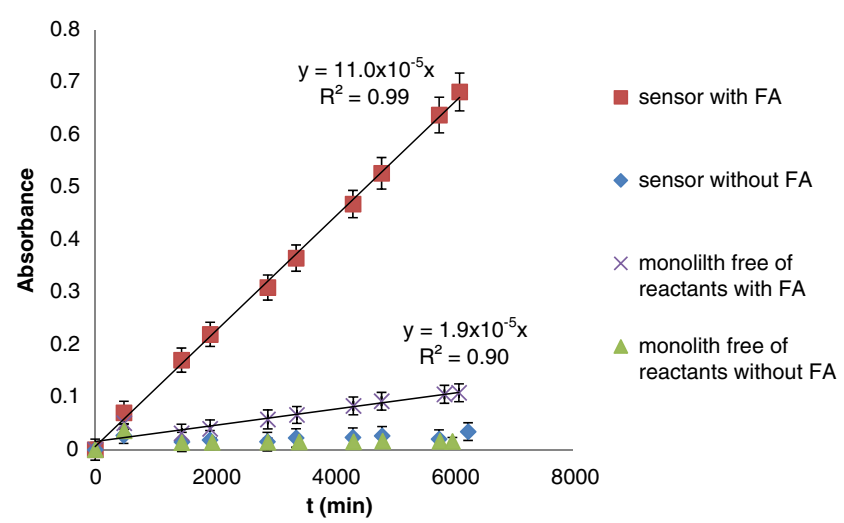

Fig. 6 Comparison of the increase in absorbance over time according to the loading of the QAI 1.4 passive sampler with a sensor or monolith free of reactants and exposure conditions (absence or presence of formaldehyde $(30 \mathrm{ppb})$ at $80 \% \mathrm{RH}$ and $20^{\circ} \mathrm{C}$ ) 
yellow-orange and blue species both present in the matrix. When the matrix is exposed to water vapour, the authors found that upon adsorption of water, the blue species diminishes gradually at the profit of the yellow-orange one and could correlate this colour change to the intrapore $\mathrm{pH}$ change from 6.5 to 5.9 . The $\mathrm{pH}$ change is slow at the beginning because the water molecules trapped in the pores have various roles: solvating first the polar BBT dye in acidic and basic forms, then acting in the deprotonation of $\mathrm{Si}-\mathrm{OH}$ and protonation of $\mathrm{Si}-\left(\mathrm{CH}_{2}\right)_{3}\left(\mathrm{NH}_{2}\right)$ and solvating the $\mathrm{Si}-\mathrm{O}^{-}$and $\mathrm{Si}-\left(\mathrm{CH}_{2}\right)_{3}\left(\mathrm{NH}_{3}\right)^{+}$species.

The ETHERA nanoporous sensor is the same TMOS/APTES (97:3 M\%) matrix but doped with Fluoral-P. When the water concentration is sufficiently high in the pores, the intrapore $\mathrm{pH}$ starts to diminish. On one hand, this $\mathrm{pH}$ drop will favour the hydrolysis of Fluoral-P, but this reaction is slow and the concentration of Fluoral-P is by far higher than that of the trapped formaldehyde. A very small decrease of Fluoral-P concentration will not affect the DDL formation. On the other hand, the $\mathrm{pH}$ drop favours the $\mathrm{pH}$-sensitive reaction of Fluoral-P with formaldehyde. Since the $\mathrm{pH}$ drop is related to the water mass transfer, $\mathrm{F}_{\mathrm{H} 2 \mathrm{O}}$, it is therefore expected that the passive samplers like QAI 1.4 and QAI 5.9 with the highest sampling rates will have the sensor responses most affected by humidity. Another important point to note is that despite the fact that the water concentration is 460 times higher than the formaldehyde concentration, formaldehyde can still diffuse into the nanoporous sensor and react with Fluoral-P. Since formaldehyde is a very polar molecule which could be solvated by water molecules in the gas phase, it is also possible that the solvated formaldehyde species is more easily adsorbed in the pores of the hydrophilic TMOS/APTES matrix.

For ETHERA sensor, the lowest impact of humidity is found for LDE 1.4 sampler which has a reduced ability to trap water $F_{\mathrm{H}_{2} \mathrm{O}}$ ) due to the reduced size of the diffusion slot $(1.4 \mathrm{~mm})$ and to a sensor position shifted apart from the slot. This last parameter appears as the most efficient feature for reducing the influence of humidity on the sensor response as shown by the comparison between the results of QAI 1.4, QAI 5.9 and QAI 1.4dep samplers. Even if the prototype LDE 1.4 appears close to the optimal version of this sampler, it could be optimised using a numerical model which was especially programmed for simulating sampling of these five prototypes [16]. In this previous article, the effective diffusion coefficients through the membrane of formaldehyde and water as well as the conversion factors between the sensor response, sampled formaldehyde mass and sampling rate were assessed and a numerical model was developed. Considering the mass transfer of water for which the sensor response is not affected by humidity (case of LDE 1.4), the geometrical characteristics of the passive sampler could be optimised.
Another possibility to diminish or remove the humidity effect while keeping a large membrane diffusion slot is to diminish the intrapore $\mathrm{pH}$ drop. This could be achieved via titrating the amount of APTES in the hybrid TMOS/APTES matrix.

\section{Conclusion}

The present work aimed at understanding the influence of humidity on the formaldehyde sensor response when using various types of passive samplers. It was demonstrated that water molecules trapped in the nanopores contribute in enhancing the reaction of the probe molecule, Fluoral-P with formaldehyde. This effect becomes important when the relative humidity is higher than $45 \%$ and is attributed to the drop of the intrapore $\mathrm{pH}$. This interference can be reduced by diminishing the water uptake rate of the sensor. This was achieved by changing the geometrical features of the passive samplers. Among the five diffusive samplers tested, the best suited was found to be LDE 1.4 which displays the smallest diffusion slot of $1.4 \mathrm{~mm}$ and a nanoporous sensor position shifted far apart from the diffusion slot.

Compliance with ethical standards The authors declare that they have no conflict of interest.

\section{References}

1. Langer S, Ramalho O, Derbez M, Ribéron J, Kirchner S, Mandin C. Indoor environmental quality in French dwellings and building characteristics. Atmos Environ. 2016;128:82-91.

2. Schauer JJ, Kleeman MJ, Cass GR, Simoneit BRT. Measurement of emissions from air pollution sources. 4. C1-C27 organic compounds from cooking with seed oils. Environ Sci Technol. 2002;36(4):567-75.

3. INERIS Fiche de données toxicologiques et environnementales des substances chimiques-Formaldéhyde, 2009.

4. Casset A, Marchand C, Purohit A, Le Calve S, Uring-Lambert B, Donnay $\mathrm{C}$, et al. Inhaled formaldehyde exposure: effect on bronchial response to mite allergen in sensitized asthma patients. Allergy. 2006;61(11):1344-50.

5. IARC. IARC Monographs on the evaluation of carcinogenic risk to humans, vol. 88. Formaldehyde, 2-butoxyethanol and 1-tertbutoxypropan-2-ol. World health Organization, International Agency for Research on Cancer; 2006. 478pp.

6. Poulhet G, Dusanter S, Crunaire S, Locoge N, Gaudion V, Merlen $\mathrm{C}$, et al. Investigation of formaldehyde sources in French schools using a passive flux sampler. Build Environ. 2014;71:111-20.

7. Wisthaler A, Apel EC, Bossmeyer J, Hansel A, Junkermann W, Koppmann R, et al. Technical Note: Intercomparison of formaldehyde measurements at the atmosphere simulation chamber SAPHIR. Atmos Chem Phys. 2008;8:2189-200.

8. W. Bell, N. Davies, K. Blakley, K. Lancaster, P. Quincey, and M. Henderson, A pilot study of formaldehyde monitoring in ambient air. NPL Rep. COEM S36, 2000. 
9. Chiappini L, Dagnelie R, Sassine M, Fuvel F, Fable S, Tran-Thi T$\mathrm{H}$, et al. Multi-tool formaldehyde measurement in simulated and real atmospheres for indoor air survey and concentration change monitoring. Air Qual Atmos Health. 2011;4(3-4):211-20.

10. Maruo YY, Nakamura J, Uchiyama M. Development of formaldehyde sensing element using porous glass impregnated with betadiketone. Talanta. 2008;74(5):1141-7.

11. Compton BJ, Purdy WC. Fluoral-P, a member of a selective family of reagents for aldehydes. Anal Chim Acta. 1980;119:349-57.

12. Tran-Thi T-H, Dagnelie R, Crunaire S, Nicole L. Optical chemical sensors based on hybrid organic-inorganic sol-gel nanoreactors. Chem Soc Rev. Feb. 2011;40(2):621-39.

13. E. Chevallier, T. Caron, C. Belon, P. Karpe, T.-H. Tran-Thi, S. Colomb, and Y. Bigay, Development of a formaldehyde chemical sensor for indoor air quantification: application in health and safety at work. Conf Vent Paris Fr. 2012.

14. Dagnelie RVH, Crassous A, Tran-Thi T-H. Scaling the hydrophobicity of porous materials to water vapour with Fluoral-P. J Memb Sci. 2010;365(1-2):98-105.

15. Paolacci H, Dagnelie R, Dominique P, Piuzzi F, Lepetit F, Tran-Thi T-H. A ppb formaldehyde gas sensor for fast indoor air quality measurements. Sensors Transducers J. 2007;82(8):1423-30.

16. Calvo-Muñoz ML, Truong TT, Tran-Thi TH. Chemical sensors of monocyclic aromatic hydrocarbons based on sol-gel materials: kinetics of trapping of the pollutants and sensitivity of the sensor. J Mater Chem. 2002;12:461-7.
17. Nash T. The colorimetric estimation of formaldehyde by means of the Hantzsch reaction. Biochem J. 1953;55(3):416-21.

18. Chevallier E, Caron T, Belon C, Karpe P, Tran-Thi T-H, Colomb S, et al. Development of a formaldehyde chemical sensor for indoor air quantification: application in health and safety at work. Paris: Vent; 2012.

19. Vignau-Laulhere J, Mocho P, Plaisance H, Raulin K, Desauziers V. Assessment of diffusion parameters of new passive samplers using optical chemical sensor for on-site measuring formaldehyde in indoor air: experimental and numerical studies. Anal Bioanal Chem. 2016;408(8):2147-57.

20. Vignau-Laulhere J, Plaisance H, Mocho P, Raulin K, Bigay Y, Desauziers V. Performance of the Radiello ${ }^{\circledR}$ diffusive sampler for formaldehyde measurement: influence of exposure conditions and ozone interference. Anal Methods. 2015;7(13):5497-503.

21. Décret no. 2011-1728 du 2 décembre 2011 relatif à la surveillance de la qualité de l'air intérieur dans certains établissements recevant du public.

22. Uchiyama $S$, Naito $S$, Matsumoto $M$, Inaba $Y$, Kunugita $N$. Improved measurement of ozone and carbonyls using a dual-bed sampling cartridge containing trans-1,2-bis(2-pyridyl) ethylene and 2,4-dinitrophenylhydrazine-impregnated silica. Anal Chem. 2009;81(15):6552-7.

23. W. Bamogo, Capteurs chimiques à base de matrices nanoporeuses pour la détection de métabolites volatils de la tuberculose. Ecole Chimie de Paris-Sud, 2015. 